\title{
Modification of DPs by epistemic adverbs*
}

\author{
Cleo Condoravdi \\ Stanford University \\ Dag Haug \\ University of Oslo
}

\author{
Mary Dalrymple \\ University of Oxford \\ Adam Przepiórkowski \\ University of Warsaw \\ Polish Academy of Sciences \\ University of Oxford
}

\begin{abstract}
We examine two phenomena which, with the exception of Bogal-Allbritten \& Weir (2017), have not been systematically studied together but are clearly related: (a) epistemic adverbs in ad-nominal positions modifying a DP outside of coordination and (b) epistemic adverbs modifying a DP within a coordination of DPs (Collins conjunction). Ad-nominal adverbs outside of coordinate structures have been claimed to have a strong reading giving rise to an existential entailment (John visited maybe England entails that John visited some place, and that place might have been England) while in Collins conjunctions, a weak reading with no existential implication has been claimed to be available (John and perhaps Mary went to the store means that either John went to the store, or John and Mary went to the store). We provide corpus data which show that weak and strong readings are available both inside and outside coordination, and we provide a unified analysis of both phenomena based in event semantics which allows modal adverbs to have sub-sentential scope and still target expressions of propositional type. Our analysis relies on the flexible approach to semantic composition afforded by glue semantics (Dalrymple 1999; Gotham 2018), where a functor can 'ignore' unsaturated positions in its arguments.
\end{abstract}

Keywords: DP modification, epistemic adverbs, adnominal adverbs, Collins conjunction

\section{Introduction}

In this paper, we look at two phenomena which, with the exception of BogalAllbritten \& Weir (2017), have not been systematically studied together but are

* Part of this work was conducted during a fellowship of the authors at the Oslo Center for Advanced Study at the Norwegian Academy of Science and Letters. We thank Dan Flickinger for many fruitful discussions at the early stages of this enterprise. We are also grateful to audiences at SPE10 in Barcelona, at the Institute of Philosophy, University of Warsaw, at Stanford University, and at SALT 29 for helpful comments. 
clearly related: epistemic adverbs in ad-nominal positions modifying a DP outside of coordination (1), and epistemic adverbs modifying a DP conjunct within a coordination of DPs (2). Following the literature, we refer to the latter case as Collins conjunction. This is a purely descriptive term and should not be taken to imply a position on whether the coordinated case is structurally different from the non-coordinated case, which is a contentious point in the literature.

(1) John visited maybe England.

(2) John and perhaps (also) Mary went to the store.

A variety of sentential adverbs, including evaluative adverbs (e.g., (un)fortunately, regrettably) and epistemic modal adverbs, can modify DPs (Ernst 1983; Huddleston \& Pullum 2002; Bogal-Allbritten 2013, 2014; Bogal-Allbritten \& Weir 2017). Ernst (1983) has argued convincingly that, syntactically, sentential adverbs can form constituents with DPs. Here we focus on epistemic modal adverbs, as they affect the at-issue content of the sentence.

Bogal-Allbritten (2013) observed that the reading of a sentence with an epistemic adverb in an ad-nominal position is stronger than the reading of the corresponding sentence with the adverb in its regular clausal position(s). For example, while (3) allows for the possibility that John visited no place, (1) does not, licencing the inferences in (3).

(3) Maybe/Possibly/Perhaps John visited England.

(4) a. John visited some place.

b. The place John visited may have been England.

That is, (1) carries an existential implication, with the uncertainty being about the witness. The same inferences arise when the modified DP is an indefinite or a definite, as in (5)-(6), which are variants of examples from Bogal-Allbritten (2013) and Ernst (1983), respectively.

(5) He got advice from perhaps a nurse.

a. He got advice from someone.

b. The person he got advice from might have been a nurse.

(6) They put their stamp of approval on perhaps the worst proposal ever submitted to the committee.

a. They put their stamp of approval on a proposal.

b. The proposal they put their stamp of approval on might have been the worst proposal ever submitted to the committee. 
Modification of DPs by epistemic adverbs

Bogal-Allbritten (2013), moreover, argued that the existential implication of epistemic adverbs in ad-nominal positions is in fact an entailment. In the context of (7), use of the epistemic adverb is felicitous both in the ad-nominal and the regular clausal position. By contrast, in the context of (8), the epistemic adverb is infelicitous in the ad-nominal position because the existential entailment conflicts with the contextually given information.

(7) Context: Mary visited Amherst yesterday and she ate at Athena's pizza. You can't remember whether Athena's or Antonio's has the most expensive pizza. You say:

a. Mary ate [ ${ }_{\mathrm{DP}}$ possibly ${ }_{\mathrm{DP}}$ the most expensive pizza in Amherst]].

b. Mary [vp possibly [vp ate the most expensive pizza in Amherst]].

(8) Context: Mary visited Amherst yesterday and planned to eat a pizza lunch at Athena's, which you know makes the most expensive pizza in Amherst. You know that Mary anticipated having to skip lunch, however, so perhaps she didn't eat anything.

a. \#Mary ate [DP possibly [DP the most expensive pizza in Amherst]].

b. Mary [vp possibly [vp ate the most expensive pizza in Amherst]].

In addition to this entailment, there are pragmatic inferences associated with epistemic adverbs in ad-nominal positions. From the utterance of (1) we get the inference that if John visited a place other than England, the speaker does not know, or is not in a position to say, what it is. Moreover, a felicitous use of a possibility epistemic modal conveys that the speaker's epistemic state allows for more possibilities than the stated one. Taken together with the existential entailment associated with the ad-nominal position of the adverb, the speaker's epistemic state is inferred to be partitioned into worlds in which John visited England and worlds in which John visited some place that was not England.

In Collins conjunctions, discussed by Collins (1988), Munn (1993), Landman (2004), Križ \& Schmitt (2012), Vicente (2013), Schein (2017), and Bogal-Allbritten \& Weir (2017), it is less clear what kind of uncertainty is communicated by epistemic adverbs. In the literature, there are arguments for both a weak and a strong reading which, in the case of (2), would license the inferences in (9) and (10), respectively.

(9) Weak reading: no existential implication

a. John went to the store.

b. Perhaps Mary also went to the store.

c. Either John or John and Mary went to the store.

(10) Strong reading: existential implication 
a. John and some other person went to the store.

b. Perhaps that other person was Mary.

Modification of DPs by epistemic adverbs raises a number of questions that have so far not received a definite answer in the literature. First of all, it is unclear what exactly an epistemic adverb modifies when it forms a constituent with a DP, since it is normally expected to target a propositional meaning. Second, there is the question of whether there is a structural distinction between ad-nominal modifiers outside of coordination and in Collins conjunction and if so, whether Collins conjunctions are ambiguous, i.e., whether the modified DP can also have the ordinary structure found outside of coordination. Related to that is the question of whether Collins conjunctions are semantically ambiguous. And if they are not ambiguous, but only have a weak reading, as many have argued, how does that square with the strong reading of ad-nominal modifiers outside of coordination? Finally, any theory of Collins conjunction must address the question of what and conjoins: is the coordination sub-clausal, or clausal with some sort of conjunction reduction?

\section{Earlier work}

Ernst (1983) was among the first to discuss ad-nominal adverbs as in (1), and to provide a series of arguments that the adverb in such examples is not just an oddly placed sentential adverb, but forms a constituent with the DP. For example, he notices that adverbs generally cannot appear between an inverted auxiliary verb and the subject, but that an adverb can modify the subject in such constructions:
a. *Will quickly Paul be here?
b. *Is usually Walter here on time?
c. Will perhaps those same hoodlums who robbed us last week be here again tonight?

This construction is also briefly discussed by Huddleston \& Pullum (2002), but neither Ernst nor Huddleston \& Pullum discuss the semantics of the construction in any detail, nor its relation to Collins conjunctions.

Much of the previous semantic literature has focused on Collins conjunctions, first discussed by Collins (1988). Notably, there has been substantial disagreement about the possible readings of such examples. Collins (1988), Landman (2004), Križ \& Schmitt (2012), Vicente (2013), and Schein (2017) recognize only a weak reading in which $A$ and possibly $B V^{\prime} d$ implies $A V^{\prime} d$ or $A$ and $B V^{\prime} d$. Schein (2017) argues against the possibility of a strong reading even in contexts that would support it: according to Schein, $A$ and possibly $B V^{\prime} d$ implies Only $A V^{\prime} d$ or $A$ and $B V^{\prime} d$. A strong reading is similarly excluded on Landman's analysis. Landman 
(2004) proposes that ad-nominal adverbials have a special interpretation, namely, as a function that acts as the identity function for some modal alternatives but maps the argument into the special null object (the bottom in the mereological boolean algebra) in other modal alternatives. Other authors recognize a strong reading for Collins conjunctions in addition to a weak reading: Munn (1993) and Bogal-Allbritten \& Weir (2017) judge that $A$ and possibly $B V^{\prime} d$ implies $A$ and someone who might have been $B V$ 'd.

Bogal-Allbritten \& Weir (2017) were the first to provide a detailed analysis of both structures and a comparison of their semantic contributions. They propose two analytic possibilities for ad-nominally modified DPs: one involves a covert relative clause-like structure and is associated with a strong reading, and the other involves clausal ellipsis and is associated with a weak reading. On both readings, the modal adverb takes sentential scope over the covert clausal material.

Building on work by Bogal-Allbritten (2013), Bogal-Allbritten \& Weir propose that ad-nominally modified DPs can appear with covert syntactic structure that is similar to a free relative clause, and that this structure can be modified by an epistemic adverb. This is possible outside coordination as well as in Collins conjuction. This structure is responsible for the strong reading with the existential entailment.

(12) Strong reading, non-coordinated DP:

a. They put their stamp of approval on perhaps the worst proposal ever submitted to the committee. $\approx$

b. ...what is perhaps the worst proposal ever submitted to the committee

(13) Strong reading, Collins conjunction:

a. John ate tiramisu and possibly the best pizza in town. $\approx$

b. ....and what is possibly the best pizza in town

Note that in the coordinate case (13b), and coordinates DPs with covert relative clause-like structure.

The second structure is special to Collins conjunctions. It results from an elliptical clausal structure in which and coordinates clauses and the epistemic adverb attaches to the second clause. This conjunction reduction structure gives rise to the weak reading with no existential entailment.

(14) Weak reading, Collins conjunction:

a. John ate tiramisu and possibly the best pizza in town. $\approx$

b. ....and he possibly ate the best pizza in town

In the next section, we provide a range of naturally occurring examples which show that non-coordinate DPs as well as Collins conjunctions can be associated with both 
weak and strong readings. Our analysis does not tie the availability of weak and strong readings to the presence or absence of coordination, but rather to the type of the matrix event and whether there are existence entailments associated with the thematic role filled by the ad-nominally modified DP.

\section{New data}

The aim of this section is to examine the empirical claims of the earlier work on ad-nominal uses of epistemic adverbs and to set the stage for the analysis.

An important assumption on which the analysis of Bogal-Allbritten \& Weir (2017) is built is that weak readings result from the conjunction reduction analysis of coordination. This predicts that such weak readings are limited to coordinate structures. However, the attested examples in (15a)-(15c) (from the iWeb corpus; https://www.english-corpora.org/iweb/; Davies 2018-) do not involve coordination which could license ellipsis and thereby clausal scope for the epistemic adverb, and yet they do not have natural paraphrases with existential entailment:

Weak readings in non-coordinate contexts:

a. Wednesday and Thursday will be a mixture of sunny spells and heavy showers with possibly some thunder and lightning.

b. Outside of possibly Murphy there is not much high-end talent in the Hurricanes prospect pool.

c. You have to chart GDX to trade NUGT, but GDX looks like 25 will be tested over the next few trading days, after perhaps a dip down, perhaps not.

For example, in the first - meteorological - example, there is no entailment that sunny spells and heavy showers must be accompanied by any other weather phenomena, such as thunder or lightning.

Another prediction of the analysis of Bogal-Allbritten \& Weir (2017) is that examples with a cumulative reading, such as (16a) below, ought to get only the strong reading. However, this example clearly has a weak reading, given in (16b). This reading cannot be derived from clausal ellipsis.

Weak reading with cumulative predicate:

a. Joe, Sue and maybe Bill (between them) ate the 5 pizzas we brought to the party.

b. The 5 pizzas we brought to the party were eaten by Joe and Sue or by Joe, Sue, and Bill.

Križ \& Schmitt (2012) noted the availability of cumulative readings for Collins 
Modification of DPs by epistemic adverbs

conjunctions and discussed its significance for potential analyses of Collins conjunctions. They also noted an important difference between Collins conjunctions and cases where a DP is added as an afterthought to a mention of a plurality, what they term 'semantic grafts': while both Collins conjunctions and semantic grafts allow for collective predicates, only Collins conjunctions are compatible with cumulative predications.

(17) Semantic grafts with collective vs. cumulative predications:

a. Joe and Sue met yesterday ... and maybe/of course also Bill.

b. \#Joe and Sue ate the 5 pizzas we brought to the party ... and maybe/of course also Bill.

Bogal-Allbritten \& Weir (2017) reconcile a conjunction reduction analysis of Collins conjunctions with the acceptability of collective predicates by essentially reducing such cases to semantic grafts involving elided clauses. If semantic grafts do not support cumulative predications, conjunction reduction cannot be the source of the weak reading of Collins conjunctions with cumulative predicates.

Hence, not only are weak readings of ad-nominal epistemic adverbs observed outside of coordination, which undermines an analysis relying on conjunction reduction, but it is also clear that not all weak readings within coordination may be explained with recourse to this kind of ellipsis.

Conversely, it is sometimes claimed that strong readings are not available within coordination. For example, Schein (2017: 43) claims that (18a) is ungrammatical because it has only the reading in (18c), and not the reading in (18b).

a. Biff and possibly Tiff met. (unacceptable according to Schein (2017): equivalent to (18c) and not (18b))

b. Biff and somebody else, possibly Tiff, met.

c. \#Biff met or possibly Biff and Tiff met.

However, the naturally occurring (19a)-(19b) show that collective predicates can support strong readings.

(19) Strong readings in coordinate contexts:

a. Ed Dickson and perhaps the recently-met with Terrelle Pryor can combine to fill Jimmy Graham's shoes. ${ }^{1}$

b. L. jourdaniana is thought to have been a cross breed between a Lophophora and possibly Turbinicarpus. ${ }^{2}$

1 https://247sports.com/nfl/seattle-seahawks/Article/Seahawks-limited-cap-space-hindering-splash-moves-116489508/

2 From the iWeb corpus. 
For example, in (19a), two people can combine to fill Jimmy Graham's shoes: Ed Dickson and somebody else, perhaps Terrelle Pryor. It should be noted that strong readings of Collins conjunctions do not necessarily result from sortal requirements of verbs (e.g., combine in (19a)) or prepositions (e.g., between in (19b)). As the following examples demonstrate, a strong reading may be forced by the context either textual, as in (20a), or non-textual, as in (20b), which is the title of a portrait of a woman and a man. ${ }^{3}$

(20) Strong readings in coordinate contexts:

a. After spending the day with Jesus, the two disciples (Andrew and possibly John the Evangelist) find Simon, saying...

b. Susan Taylor Wilson and possibly John Wilson

Collins (1988: 10) argues against encoding a strong reading into the semantics, based on the infelicity of both: if the semantics of (21a) made available a strong reading, that would guarantee that the presupposition of both for a doubleton domain is satisfied, and hence (21b) would be felicitous. However, there is a clear contrast between (21b) and (21c).

(21) a. John and perhaps Mary ran.

b. \#Both John and perhaps Mary ran.

c. Both John and some girl, who was perhaps Mary, ran.

Even if the semantics does not directly make available a strong reading for Collins conjunctions, the question arises why the presupposition of both cannot be accommodated in cases like (21b), with the speaker being understood as conveying uncertainty about the identity of the second individual. This is arguably how the sortal requirement of collective predicates for a group argument can be satisfied in cases like (19), with the strong reading arising in context. We hypothesize that the contrast between (21b) and (21c) is due to the fact that - as argued by Hendriks (2004) - both in such constructions is a focus particle, similar to such additive focus particles as also and too:
a. John ate both the rice and the beans.
b. John ate the rice and also the beans.
c. John ate the rice and the beans too.

According to Hendriks (2004: 125-132), from which the above examples are taken,

3 (20a) is taken from https://www.catholicworldreport.com/2018/01/13/ christs-question-for-everyone-what-are-you-looking-for/, and (20b) from https://www. digitalcommonwealth.org/search/commonwealth:ks65hm080?view=commonwealth\%

3Aks65hm119 
Modification of DPs by epistemic adverbs

the additive focus in $(22 \mathrm{~b}-\mathrm{c})$ - with also and too - is the second conjunct, the beans, while the first conjunct, the rice, constitutes the explicitly given singleton set of alternatives. Similarly, in (22a) - which involves the focus particle both - the additive focus is the first conjunct, the rice, while the second conjunct, the beans, is the explicit alternative. If so, it is natural to assume that the set of alternatives in such focus constructions must be discourse-given, resulting in all three focus particles being associated with anaphoric presuppositions, which cannot be accommodated. As is by now well known, also and too are associated with anaphoric, rather than simply existential, presuppositions (Kripke 2009; Heim 1992). If this is on the right track, we can make sense of the contrast between (21b) and (21c), while still allowing for strong readings of Collins conjunctions to arise in context.

\section{Analysis}

The idea behind the analysis we pursue is that modal adverbs that attach to DPs have sub-sentential scope but still target expressions of propositional type. We use event semantics in order to have predicates and their dependents compose via thematic roles. This allows the epistemic adverb to combine with a DP and have semantic scope just over the thematic role linking the DP to the verb, rather than over the entire predication. On our analysis, then, the uncertainty conveyed by the use of the ad-nominal epistemic adverb is ultimately about the relevant thematic role. However, the existential quantification over the event scopes over the epistemic adverb, so that the existence of an event of a particular type $i$ s asserted. If the event is of a type that entails a particular thematic role, an existential implication arises. Within conjunction, the modal takes scope over the specification of a DP meaning as constituting a part of the entity that is the value of a thematic role.

It is crucial for our analysis that a propositional modifier (i.e., something of type $\langle t, t\rangle$ or the corresponding intensional type) can target not just something of type $t$, but any boolean type, i.e., any type ending in $t$. In particular, the adverb combines not with a proposition but with a property of events (type $\langle v, t\rangle$ ). For this to work, we need a flexible system of semantic composition where we can delay saturation of the event variable and combine with the underlying type $t$ object. To achieve that we use glue semantics (Dalrymple 1999; Gotham 2018), where a functor can 'ignore' unsaturated positions in its arguments. 


\subsection{The framework}

\subsubsection{Some background on glue semantics}

In glue semantics, syntax does not specify a semantic composition tree directly, but only types. Base types are of the form $e_{n}$ and $t_{n}$, where $n$ is a syntactic index and $e$ and $t$ are type constructors corresponding to Montague's types $e$ and $t$. Complex types are formed with linear implication $\multimap$ (corresponding to lambda abstraction). These types can be assembled in a linear logic proof of $t_{r}$ (where $r$ is the index of the root) and the meanings are assembled accordingly. The two proof rules of (the relevant fragment of) linear logic are implication introduction and elimination. Implication elimination corresponds to functional application (23).

Implication elimination/function application:

$$
\frac{\text { jump }: e_{1} \multimap t_{2} \quad \text { john }: e_{1}}{j u m p(j o h n): t_{2}}
$$

Implication introduction corresponds to lambda abstraction (24).

Implication introduction/lambda abstraction:

$$
\begin{gathered}
{\left[\mathbf{x}: e_{1}\right]} \\
\vdots \\
\frac{P(\mathbf{x}): t_{2}}{\lambda x \cdot P(x): e_{1} \multimap t_{2}}
\end{gathered}
$$

This allows for a number of important flexible composition techniques, including (temporarily) skipping an unsaturated argument to deal with quantifiers in object position (25), and type raising (26).

(25) John saw everything:

$$
\begin{aligned}
& \frac{\lambda x . \lambda y \cdot \operatorname{see}(x, y): e_{1} \multimap e_{2} \multimap t_{3} \quad\left[\mathbf{x}: e_{1}\right]}{\lambda y . \operatorname{see}(\mathbf{x}, y): e_{2} \multimap t_{3}} \quad \lambda P . \forall x . P(x):\left(e_{2} \multimap t_{3}\right) \multimap t_{3} \\
& \forall y \cdot \operatorname{see}(\mathbf{x}, y): t_{3} \\
& \frac{\overline{\lambda x . \forall y . \operatorname{see}(x, y): e_{1} \multimap t_{3}} \quad \text { john }: e_{1}}{\forall y \cdot \operatorname{see}(j o h n, y): t_{3}}
\end{aligned}
$$

(26) Type-raising: 
Modification of DPs by epistemic adverbs

$$
\frac{\frac{j o h n: e_{1} \quad\left[\mathbf{P}: e_{1} \multimap t_{2}\right]}{P(j o h n): t_{2}}}{\lambda P . P(j o h n):\left(e_{1} \multimap t_{2}\right) \multimap t_{2}}
$$

\subsubsection{Coupling glue semantics with event semantics}

A standard assumption in neo-Davidsonian event semantics is that thematic roles (and prepositions) turn DPs into modifiers of verbal projections. Following Champollion (2015), we assume that this happens low in the semantic composition tree. Since we are not dealing with irreducibly quantificational DPs, we can assume that dependents combine with event predicates intersectively. ${ }^{4}$ This means that we treat thematic roles as functions from events to individuals.

Let us first see how this works for a simple example without an epistemic adverb. We start with the lowest type possible for a DP, using a choice function analysis for the indefinite in (27).

$$
\mathrm{John}_{1} \mathrm{saw}_{2} \text { a nurse } 3 .
$$

The lexical meanings are given in (28). The index on the types is determined by the syntactic role of the given expression, and the types determine what can compose with what.

$$
\begin{array}{lll}
\text { John } & j o h n & e_{1} \\
\text { a nurse } & f(\text { nurse }) & e_{3} \\
\text { see } & \lambda \text { e.see }(e) & v_{2} \multimap t_{2} \\
\text { theme } & \lambda \text { e. } \lambda x . \operatorname{th}(e)=x & v_{2} \multimap e_{3} \multimap t_{3} \\
\text { agent } & \lambda \text { e. } \lambda x \cdot a g(e)=x & v_{2} \multimap e_{1} \multimap t_{1} \\
\text { intersect } & \lambda P . \lambda Q . \lambda e . P(e) \wedge Q(e) & \forall x . \forall y . \forall z \cdot\left(v_{x} \multimap t_{y}\right) \multimap\left(v_{x} \multimap t_{z}\right) \multimap v_{x} \multimap t_{z}
\end{array}
$$

Notice that glue semantics is strictly based on function application. This means that we cannot simply introduce composition principles like predicate modification (Heim \& Kratzer 1998: 65). Instead, we encode such principles as semantic resources, in this case intersect, which has the same effect as predicate modification. We assume that the semantic contributions encoding principles of semantic composition, including intersect as well as the bind operator introduced in the next section, can be applied whenever needed, and are not subject to the principles of resource accounting which are standardly assumed for lexically contributed premises in a glue setting. (29) shows how the glue composition proceeds.

4 To deal with quantificational DPs we would adopt quantifiers over events and lift thematic roles. 


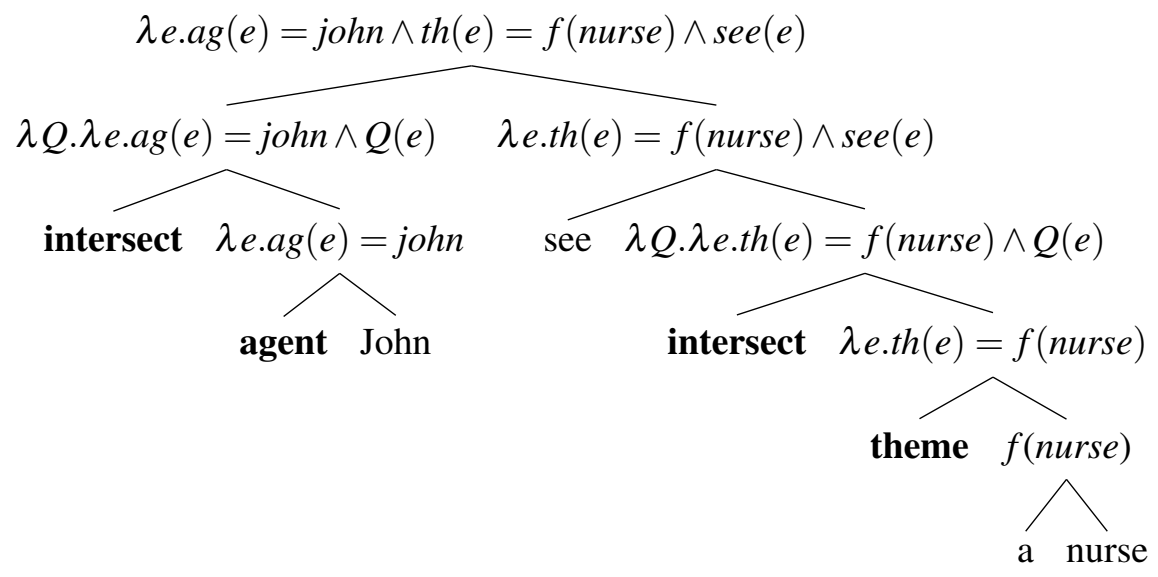

In a coordinate structure of DPs, and operates on DP meanings. (30) gives some sample meanings. Given the meaning of and, DPs need to be type raised. As was shown in (26), this comes for free in glue semantics. Notice that we assume that conjunction is binary and that $A, B$ and $C=A$ and $B$ and $C$.

$$
\begin{array}{ll}
\text { John } & \lambda P . P(\text { john }) \\
\text { a nurse } & \lambda P . P(f(\text { nurse })) \\
\text { and } & \lambda \mathscr{Q}_{1} \cdot \lambda \mathscr{Q}_{2} \cdot \lambda P . \mathscr{Q}_{1}(\lambda y \cdot y \sqsubseteq \oplus P) \wedge \mathscr{Q}_{2}(\lambda y \cdot y \sqsubseteq \oplus P)
\end{array}
$$

(32) shows how the composition goes for (31a) given these meanings.

a. John and a nurse met.

b. \#John met.

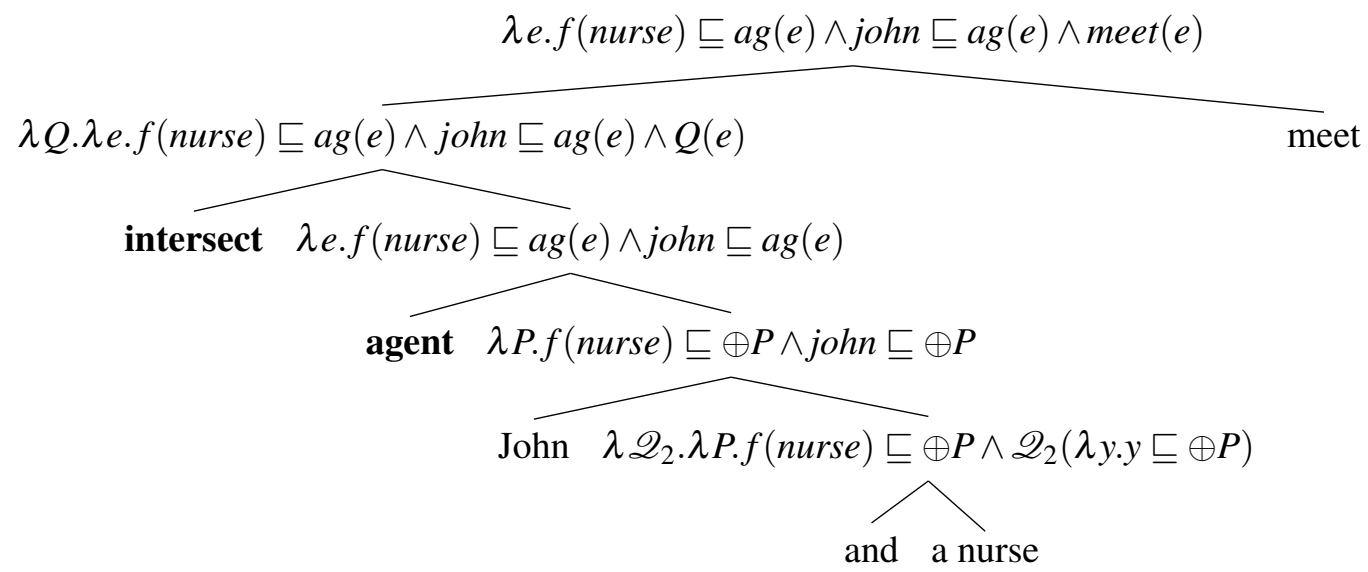

We rely on the functionality of thematic roles to simplify $\oplus a g(e)$ as just $a g(e)$. On our analysis, the contribution of and results in an asymmetry between non-conjoined and conjoined DPs: a non-conjoined DP exhausts its corresponding thematic role, 
Modification of DPs by epistemic adverbs

but conjoined DPs need not exhaust the thematic role. This accounts for the infelicity of (31b).

On the other hand, since our analysis requires group formation through and to be unbounded, the semantics of (31a) allows for someone else, in addition to John and the given nurse, to have participated in the meeting. We have to assume that, one way or another, implicit exhaustification negates non-weaker alternatives, resulting in the strengthened meaning that excludes any other individuals from being part of the agent of the meeting event. Exhaustification could be part of closing off the list of conjuncts in a conjunction. ${ }^{5}$ Although we do not want to commit ourselves to a particular view of exhaustification and how it comes about, we are committed to a specific stance on how the negated alternatives are determined, since what needs to be excluded are conjunctions with a longer list of conjuncts. That is, we must assume that propositions of the form " $a$ and $b$ P'ed" generate an alternative like " $a$ and $b$ and someone else $P$ 'ed", which is negated by exhaustification.

\subsection{Analysis of ad-nominal epistemic adverbs}

To deal with epistemic adverbs, we need to intensionalize our system. This could be done in several ways; we opt here for introducing explicit world variables in the semantics and a corresponding type constructor $s$ on the glue side. Ultimately these world variables will be bound by intensional operators, and we can use glue to delay saturation until we meet such an operator. However, at that point there will typically be several unsaturated world arguments, and we use a bind operator to identify them. Notice that this makes it possible to delay saturation of a world variable across a modal operator and bind it at a later stage to get a de re reading. As such, the system is similar to the unconstrained binding of world pronouns explored in von Fintel \& Heim (2011: chapter 8), and it will need to be constrained by a binding theory for world variables in the same way, although that is not relevant here.

(34) gives the lexical entries we need for (33).

(33) $\mathrm{John}_{1} \mathrm{saw}_{2}$ perhaps a nurse 3 .

5 Consider, for instance, Zimmermann's (2000) closure condition on a list of possibilities, which derives the exhaustivity of disjunction. 
Condoravdi, Dalrymple, Haug, and Przepiórkowski

$$
\begin{array}{lll}
\text { John } & \lambda w \cdot \text { john } & s_{1} \multimap e_{1} \\
\text { a nurse } & \lambda w \cdot f\left(\text { nurse }_{w}\right) & s_{3} \multimap e_{3} \\
\text { see } & \lambda w \cdot \lambda{\text { e. } \operatorname{see}_{w}(e)}(e) v_{2} \multimap t_{2} \\
\text { theme } & \lambda w \cdot \lambda \text { e. } \lambda x \cdot t h_{w}(e)=x & s_{3} \multimap v_{2} \multimap e_{3} \multimap t_{3} \\
\text { agent } & \lambda w \cdot \lambda e \cdot \lambda x \cdot a g_{w}(e)=x & s_{1} \multimap v_{2} \multimap e_{1} \multimap t_{1} \\
\text { intersect } & \lambda P \cdot \lambda Q \cdot \lambda e \cdot P(e) \wedge Q(e) & \forall x \cdot \forall y \cdot \forall z \cdot\left(v_{x} \multimap t_{y}\right) \multimap\left(v_{x} \multimap t_{z}\right) \multimap v_{x} \multimap t_{z} \\
\text { perhaps } & \lambda p \cdot \lambda w \cdot \nabla_{w} p & \left(s_{3} \multimap t_{3}\right) s_{3} \multimap t_{3} \\
\text { bind } & \lambda P \cdot \lambda w \cdot P(w)(w) & \forall x \cdot \forall y \cdot\left(s_{y} \multimap s_{x} \multimap t_{y}\right) \multimap s_{y} \multimap t_{y}
\end{array}
$$

The modal adverb targets a meaning contribution of type $s_{3} \multimap t_{3}$. In the case of ad-nominal modification outside of conjunction, as we have here, a $s_{3} \multimap t_{3}$-type contribution is provided when a nurse combines with its thematic role theme. (35) shows how the semantic derivation goes for see perhaps a nurse ( $w^{\prime \prime}$ and $w^{\prime}$ are eventually identified by bind applying at the root level, not shown here); bind under perhaps does not contribute any meaning, but simply identifies two world variables:

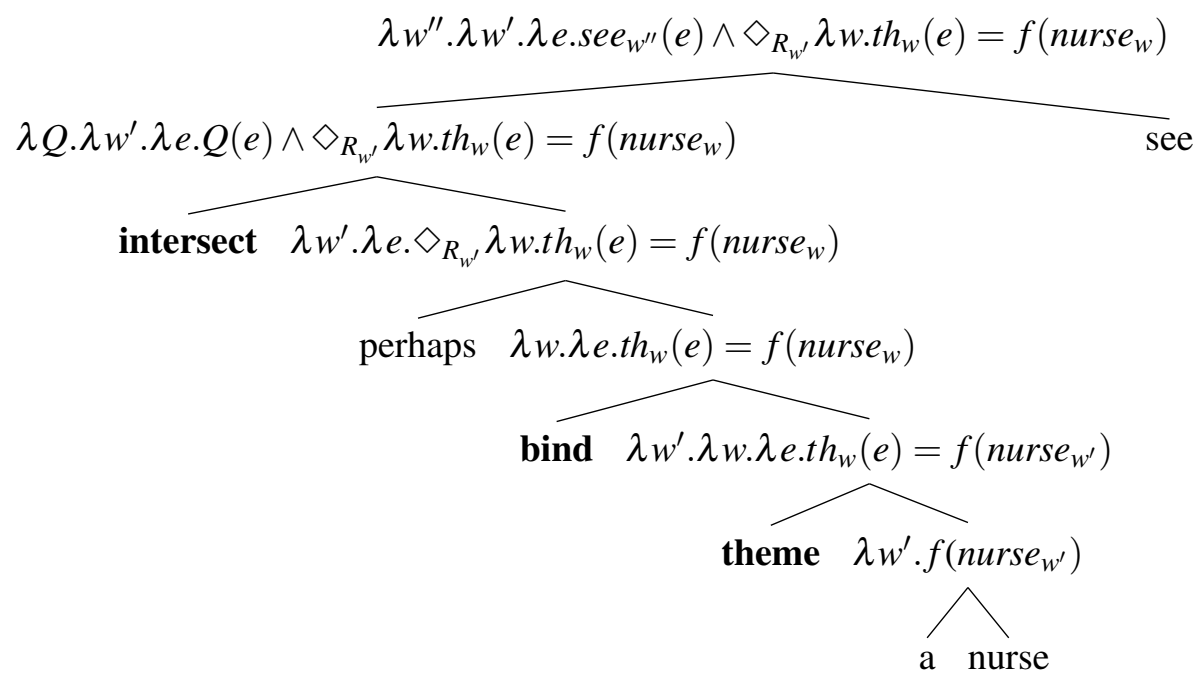

Notice that we are crucially relying on delayed saturation. First, the world argument of a nurse is temporarily ignored during combination with theme; at a later stage, the event argument introduced by theme is temporarily ignored by perhaps.

When the DP is a definite description, the semantic derivation is exactly parallel, as shown in (36) (for approved perhaps the proposal...). 
Modification of DPs by epistemic adverbs

$$
\lambda w^{\prime \prime} . \lambda w^{\prime} . \lambda \text { e.approve }_{w^{\prime \prime}}(e) \wedge \diamond_{R_{w^{\prime}}} \lambda w \cdot t_{w}(e)=\text { xx.proposal }_{w}(x)
$$

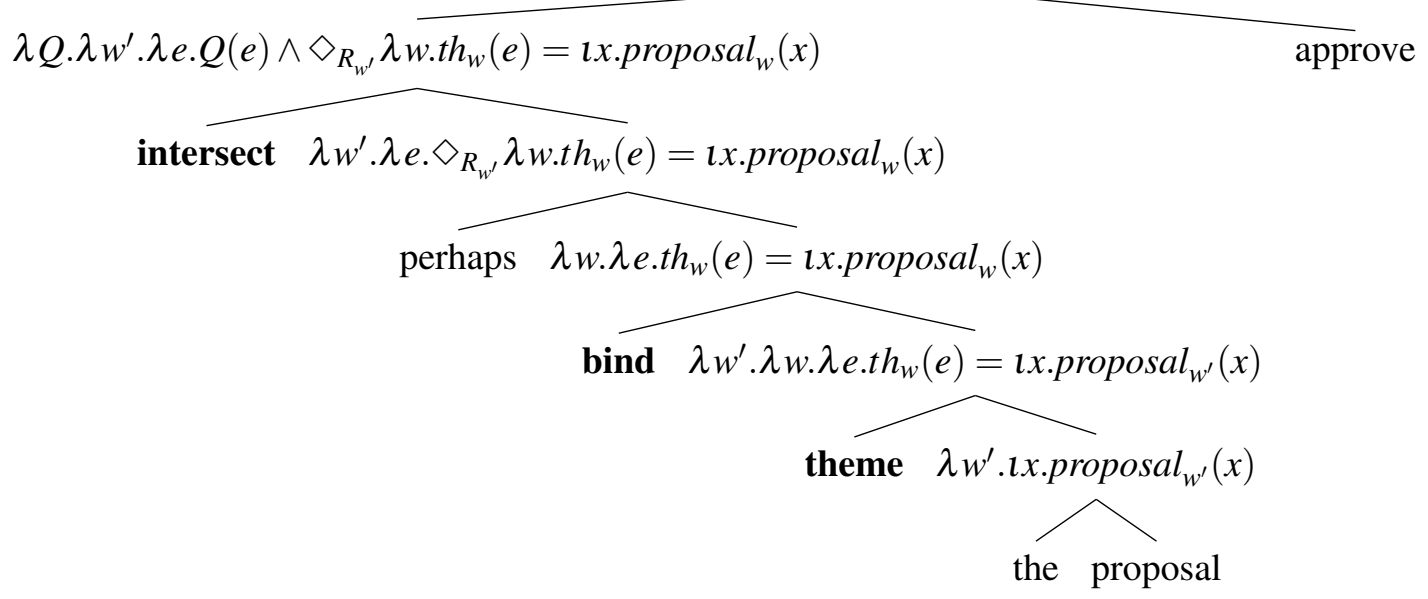

In fact, there are many ways the speaker can be uncertain about the thematic role in such cases. Consider (37).

a. We put our stamp of approval on perhaps the worst proposal ever submitted to the committee.

b. They put their stamp of approval on perhaps the worst proposal ever submitted to the committee.

In the first person example (37a), it is natural to infer that the speaker knows which proposal was approved, and she can then be taken to be uncertain about which among the proposals submitted to the committee was in fact the worst one. In the third person example (37b), we can infer that the speaker knows which proposal was the worst one, but is uncertain about which proposal was approved. In general, the speaker can also be inferred to be uncertain about both, and in that case (37b) just conveys the possibility that the proposal approved and the worst proposal were the same.

Prepositions can also introduce thematic roles and compose with a DP to produce a meaning which the modal adverbial can then apply to. This is shown in (38) for with perhaps a knife. Notice that in this case, the semantic composition tree does not match the surface syntax. Because of its type, perhaps cannot combine with $a$ knife, which is of type $e$, before the latter has combined with its thematic role. 


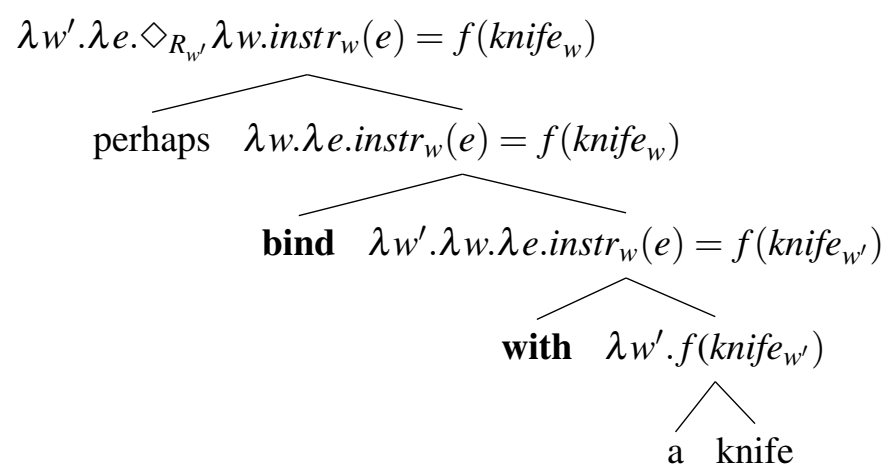

In general, our analysis predicts that when perhaps scopes over the DP, the uncertainty is about the identity of some thematic role, whereas the event itself is independently asserted to exist. This means that when the type of event entails the existence of a participant bearing a thematic role, that thematic role is inferred to be defined across the epistemic state of the speaker. In such cases, the uncertainty is about what (kind of) individual bears the relevant thematic role: the stated one or someone/thing else. When the type of event does not entail the existence of a participant bearing a thematic role, then that thematic role is inferred to be defined in some worlds of the epistemic state of the speaker and undefined in others, which means that the uncertainty is about whether any individual bears the relevant thematic role: the stated one or no one/nothing. In other words, we predict that we get existential entailment (outside of coordination) in those cases where the event entails a particular thematic role, as shown in (39)-(40).

(39) John stabbed Bill with possibly an icepick. uncertainty about the kind of instrument used in the stabbing

(40) John and Bill fought with possibly an icepick. uncertainty about whether a weapon was used in the fight

No earlier analysis predicts this pattern.

\subsection{Analysis of Collins conjunction}

Within a coordinate structure, the modal adverb composes directly with the modified DP before application of the thematic role. Given the meaning of and that we saw in (30), a DP must be type raised before it combines with and. By type raising, it gets a type that the adverb can combine with. The thematic role is filled in once the entire conjunction is assembled. (41) shows how this works.

John and perhaps Mary left. 
Modification of DPs by epistemic adverbs

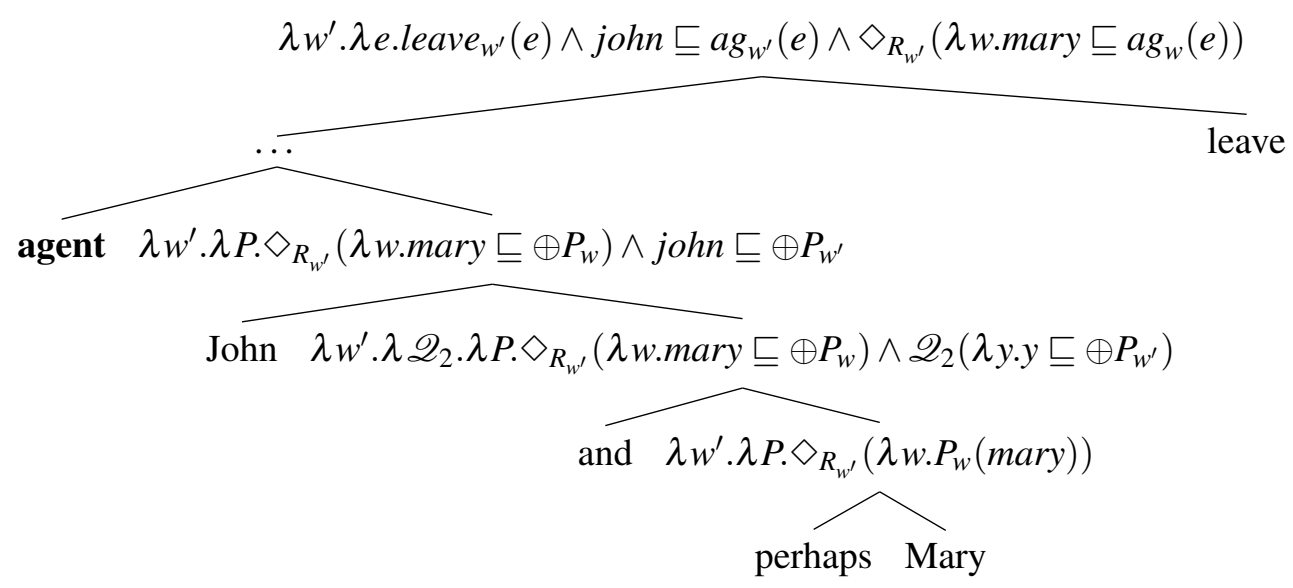

It is useful to look at what happens when perhaps and Mary combine: in the type raised representation of Mary, $\lambda w . \lambda P . P_{w}$ (mary), we temporarily ignore the $P$ to yield $\lambda w . \mathbf{P}_{w}$ (mary); next we apply perhaps and abstract over $P$ again to yield $\lambda w^{\prime} . \lambda P . \diamond_{R_{w^{\prime}}}\left(\lambda w . P_{w}(\right.$ mary $\left.)\right)$, i.e., a function from evaluation worlds to the set of properties such that Mary has them in some world accessible from the evaluation world.

In the next step, and combines this and the type-raised version of john. Finally, agent saturates the property argument. When we combine this with leave, we get a set of worlds $w^{\prime}$ and events $e$ such that $e$ is a leaving event (in $w^{\prime}$ ), John is part of the agent of $e$ in $w^{\prime}$, and as far as the speaker knows in $w^{\prime}$, Mary may have been part of the agent of $e$. To this we apply existential closure of the event variable.

Unlike with ad-nominal modification outside of conjunction, one of the conjuncts can always provide a value for a given thematic role. This means that our semantics predicts that, in general, the weak reading arises in Collins conjunctions. In (41), even if we assert (after existential closure) that there is a leaving event, we also assert that John was part of the agent of that event, and this is enough to satisfy the entailment associated with a leaving event and there is no entailment that someone other than John also left. Therefore, we get the reading that either John left, or John and Mary left; more generally, $A$ and possibly $B V^{\prime} d$ implies $A V^{\prime} d$ or $A$ and $B V^{\prime} d$. As mentioned earlier, this account of Collins conjunction needs to be supplemented with an account of the exhaustivity of conjunction in general.

Although we predict that the weak reading arises in the general case of Collins conjunction, the strong reading can arise if the context supports it. For example, if the context makes it clear that the speaker believes that two individuals were involved in some event, it is natural to take the speaker's uncertainty to be about the identity of the second individual. Moreover, our semantics predicts that the strong reading arises due to the verbal predicate in a special case: when the predicate is 
collective and the unmodalized conjunct is singular. This happens in (18a) above, which, on our analysis, is equivalent to (18b), not (18c).

\section{Conclusion}

We have provided a unified analyis of ad-nominal modifiers outside of coordination and in Collins conjunctions, relying on the flexible composition techniques afforded by glue semantics to allow epistemic modifers to take non-clausal scope. Our analysis explains the generally strong reading of ad-nominal modifiers outside of coordination and the generally weak reading in Collins conjunctions, but allows for both weak and strong readings in both environments.

\section{References}

Bogal-Allbritten, Elizabeth. 2013. Modification of DPs by epistemic modal adverbs. In Maria Aloni, Michael Franke \& Floris Roelofsen (eds.), Nineteenth Amsterdam Colloquium, 51-58. Amsterdam: Institute for Logic, Language, and Information, University of Amsterdam.

Bogal-Allbritten, Elizabeth. 2014. Interpreting DP-modifying modal adverbs. Handout of talk presented at SALT 2014. http://www.nyu.edu/projects/salt2014/ SALT_2014/Program_files/ModalAdverbs.pdf.

Bogal-Allbritten, Elizabeth \& Andrew Weir. 2017. Sentential and possibly subsentential modification: The ambiguity of Collins conjunctions. In Andrew Lamont \& Katerina Tetzloff (eds.), NELS 47, 89-102.

Champollion, Lucas. 2015. The interaction of compositional semantics and event semantics. Linguistics and Philosophy 38. 31-66.

Collins, Chris. 1988. Part I: Conjunction adverbs. Unpublished manuscript, MIT.

Dalrymple, Mary (ed.). 1999. Semantics and syntax in Lexical Functional Grammar: The resource logic approach. Cambridge, MA: The MIT Press.

Davies, Mark. 2018-. The 14 billion word iWeb corpus. http://corpus.byu.edu/iWeb/.

Ernst, Thomas. 1983. Towards an integrated theory of adverb position in English: Indiana University $\mathrm{PhD}$ dissertation.

von Fintel, Kai \& Irene Heim. 2011. Intensional semantics. http://web.mit.edu/ fintel/fintel-heim-intensional.pdf.

Gotham, Matthew. 2018. Making Logical Form type-logical: Glue semantics for Minimalist syntax. Linguistics and Philosophy 41(5). 511-556.

Heim, Irene. 1992. Presupposition projection and the semantics of attitude verbs. Journal of Semantics 9(3). 183-221.

Heim, Irene \& Angelika Kratzer. 1998. Semantics in generative grammar. Malden, MA: Blackwell. 
Modification of DPs by epistemic adverbs

Hendriks, Petra. 2004. Either, both and neither in coordinate structures. In Alice ter Meulen \& Werner Abraham (eds.), The composition of meaning: From lexeme to discourse Amsterdam, 115-138. John Benjamins.

Huddleston, Rodney \& Geoffrey K. Pullum. 2002. The Cambridge grammar of the English language. Cambridge, UK: Cambridge University Press. doi:https://doi.org/10.1017/9781316423530.

Kripke, Saul A. 2009. Presupposition and anaphora: Remarks on the formulation of the projection problem. Linguistic Inquiry 40(3). 367-386.

Križ, Manuel \& Viola Schmitt. 2012. Adverbial conjunctions: Exposition of a problem. Unpublished manuscript. http://homepage.univie.ac.at/viola.schmitt/ kriz_schmitt_2012.pdf.

Landman, Fred. 2004. Indefinites and the type of sets (Explorations in Semantics 3). Oxford: Blackwell.

Munn, Alan Boag. 1993. Topics in the syntax and semantics of coordinate structures: University of Maryland PhD dissertation.

Schein, Barry. 2017. 'And': Conjunction reduction redux. Cambridge, MA: The MIT Press. doi:https://doi.org/10.7551/mitpress/10488.001.0001.

Vicente, Luis. 2013. In search of a missing clause. Handout of a talk given at Universität Potsdam.

Zimmermann, Thomas Ede. 2000. Free choice disjunction and epistemic possibility. Natural Language Semantics 8. 255-290.

Cleo Condoravdi

Department of Linguistics

Stanford University

Stanford, CA 94305-2150

USA

cleoc@stanford.edu

Dag Haug

Department of Linguistics and Scandinavian Studies

PO Box 1102 Blindern

0317 Oslo

Norway

daghaug@ifikk.uio.no
Mary Dalrymple

Centre for Linguistics and Philology

Walton Street

Oxford OX1 2HG

United Kingdom

mary.dalrymple@ling-phil.ox.ac.uk

Adam Przepiórkowski

IPI PAN

ul. Jana Kazimierza 5

01-248 Warszawa

Poland

adamp@ipipan.waw.pl 